\title{
REINCIDENCIA: CRÍTICA METODOLÓGICA Y PROPUESTA DE MEDICIÓN E INTERPRETACIÓN PARA EL SISTEMA PENAL CHILENO
}

\author{
ANDRÉS AEDO HENRÍQUEZ*
}

\begin{abstract}
RESUMEN: El presente artículo tiene por objetivo ofrecer una visión metodológica de la/s reincidencia/s, ya que el nivel de confusión respecto del término, tanto jurídica como metodológicamente, es equivalente a su uso intensivo por parte de los actores del sistema político y de los medios de comunicación. Este estudio procura mostrar que la reincidencia puede ser medida de varias formas, y que algunas de estas metodologías tienen la capacidad de constituirse en fuente de información relevante para las instituciones que manejan este concepto de manera técnica, porque les sirve de indicador primordial para su labor. En el caso de Gendarmería de Chile, si bien no es el principal indicador, tiene directa relación con su quehacer, toda vez que estas cifras hacen parte de los procesos normales de autoobservación institucional.

Las cifras presentadas han sido extraídas de diferentes fuentes, incluyendo estudios realizados por Gendarmería a través de distintas instancias. El énfasis es, entonces, metodológico, presentado tasa por tasa, tratando de poder revelar los elementos de validez de su interpretación y cálculo. La estandarización posible de las tasas de reincidencia, por medio de la crítica de investigaciones anteriores, representa el objetivo central de este trabajo.
\end{abstract}

Palabras clave: sistema penal, reincidencia, metodología de medición.

ABSTRACT: The article in question aims to offer a methodological view of reoffending, as the level of confusion on the concept, legally and methodologically, is equivalent to its intensive use by actors in the political system and the media. This study tries to show that reoffending can be measured in different ways, and that some of these methodologies have the capacity of becoming a source of relevant information for the institutions that deal with this concept in a technical way, as it is useful as a main indicator for their tasks. In the case of the Prison Police Service of Chile (Gendarmería de Chile), although it is not the main indicator, it has direct relation with its affairs, as this data is part of the normal processes of institutional self observance. The data presented have come from different sources, including studies carried out by Prison Police Service of Chile in various instances. It is then a methodological approach, presenting rate by rate, trying to reveal the elements of validity of interpretation and calculation. The possible standardization of the rates of reoffending, through the critical analysis of previous research, is the central aim of this article.

Key words: criminal System, reoffending, measurement methodology.

"Y se ve que detrás del llamado telón, que debe cubrir el interior, no hay nada que ver a menos que penetremos nosotros mismos tras él, tanto para ver,como para que haya detrás algo que pueda ser visto". G.W.F. Hegel. Fenomenología del espíritu.

"...todas las teorias nacen refutadas y mueren refutadas" Imre Lakatos. La metodología de los programas de investigación científica.

* El autor es Antropólogo Social y (C) Magíster en Sociología de la Modernización por la Universidad de Chile. Se desempeña como profesor del Departamento de Antropología de la Universidad de Chile e investigador de la Unidad de Investigación Criminología de Gendarmería de Chile. andres.aedo@gendarmeria.cl 


\section{INTRODUCCIÓN}

La reincidencia en Chile ha llegado a ser uno de los temas más debatidos, en cuanto a su condición de agravante penal, en el conjunto de instituciones dedicadas al estudio y a la generación de iniciativas de política criminal. Sin embargo, no hay un acuerdo general, respecto de qué representa el concepto, cómo vamos a medirlo, cuál es y será su nuevo peso penal y su actuación de indicador de gestión de la política criminal o de reinserción social. Uno de los elementos interesantes y que respaldan esta afirmación es que los trabajos científicos sobre el proceso de reincidencia no son muy numerosos, por lo mismo no hay un acabado estudio del fenómeno, y las políticas públicas han avanzado en base a percepciones derivadas de posiciones político-electorales antes que en estudios empíricos. De esta manera, se van presentado limitaciones teóricas, empíricas e interpretativas sobre el estudio de la reincidencia.

Una primera limitación teórica es el nivel de polisemia del concepto, el cual es tan profundo que podemos nombrar una serie de conceptos asociados a este, como: reiteración, reencarcelamiento, reincidencia criminógena, reincidencia empírica, reincidencia jurídica, reincidencia genérica, específica, propia e impropia; sin que se haya fijado alguno de ellos como el indicador que empleará el sistema de justicia para poder cuantificar el fenómeno. Una segunda limitante teórica consiste en que cada uno de estos conceptos presenta matices sobre el problema que están tratando de abordar, donde se produce una confrontación entre elementos propios del derecho y de las ciencias sociales; por ejemplo si se fija el problema en la socialización delictual por el internamiento penal, el reencarcelamiento y la reincidencia legal son similares en sus efectos sobre la subjetividad de los internos; pero si se fija desde un punto de vista jurídico resulta un abismo. Una tercera limitación teórica es el nulo miramiento sobre los elementos sociales que confluyen en el fenómeno delictivo y en la reincidencia, considerando a esta solo como un elemento individual de falta de apego a las normas, desconociéndose así, casi un siglo de debate criminológico.

Las limitaciones empíricas y metodológicas están derivadas de las anteriores; por ejemplo, no hay muchas investigaciones científicas sobre el proceso de reincidencia, aún menos estudios de corte longitudinal que muestren su evolución y/o efectividad de las políticas de control social o de las políticas de reinserción social en Chile. Por lo tanto, es posible afirmar que comenzar a debatir es la primera exigencia; un segundo paso es apoyar las opiniones sobre datos fiables y un tercero, la posibilidad de saber efectivamente qué ocurre con los sujetos al salir del sistema penal y cuáles son las condicionantes de su reingreso. Y si se me permite una última observación, es necesario también generar y aplicar políticas, pero claro, este es un modelo donde existe un proceso científico o por lo menos racional. De esta manera, como un aporte a este proceso de debate, pretendemos contribuir a propiciar una discusión sobre la reincidencia desde el sistema penal, pondremos los resultados de diferentes estudios de reincidencia realizados por Gendarmería, y extraeremos las consecuencias que de ellos se desprenden para la comprensión adecuada del fenómeno. 


\section{EL PROBLEMA CONCEPTUAL DE LA REINCIDENCIA}

El fenómeno de la inseguridad pública de los últimos 15 años ha vuelto los ojos al sistema penal, después de una década de iniciativas que se centraron en el control espacialurbano de la actividad delictual en base a la teoría situacional del delito. La cantidad de comunicaciones sociales sobre el sistema penal, como programas de televisión y reportajes de periódicos, han tendido a multiplicarse en los últimos años, abriendo un nuevo foco de la inseguridad ciudadana. El sistema penal, al interior del complejo de la inseguridad pública, se conectó con la preocupación del control social por medio de la administración institucional de las penas. Este fenómeno de relevancia internacional, donde todos los sistemas penales han tendido al aumento de la población bajo su custodia, ha llevado a este a cumplir nuevas funciones. El sistema penal se encuentra hoy atrapado entre los sistemas de la seguridad y de la justicia, donde el sistema de seguridad exige protección social, entendiendo esto como castigo de aquellos que se salen de las normas mediante el encierro institucional, mientras el sistema de justicia demanda condiciones para los internos en referencia a los derechos humanos. Ejemplos del primero son las discusiones sobre el uso de la detención preventiva; ejemplo de la segunda exigencia es la propuesta de una ley de ejecución penal. Por lo tanto, el sistema penal se encuentra en una nueva complejidad que va más allá de sus funciones institucionales normales. En el caso de la reincidencia, sin aclarar aún el contenido específico del concepto, es donde ambas exigencias muestran no solamente su presencia, sino una tensión, ya que el problema se vuelve aún más complejo. De esta forma, el trabajo intrapenitenciario con los internos deviene en un elemento fundamental en la prevención de conductas delictivas posteriores a su egreso del sistema penal.

La reincidencia es una de las nociones más utilizadas por el sistema político en cuanto a sus comunicaciones sobre la seguridad ciudadana, aunque se sabe bastante poco de cuáles son sus reales dimensiones e incidencias. La reincidencia, como mínimo, remite a la repetición de una acción delictiva de una persona; un punto central respecto a esta cuestión es que solo es reincidencia en el momento en que el Estado tiene plena certeza de que la acción desviada y penalizada por el derecho se ha realizado dos veces, esto es: a) cuando se prueba el daño de un bien jurídico, y b) cuando se prueba el dolo del victimario por medio de un procedimiento judicial. Este conjunto de elementos solo se cumple en el instante en que el victimario es penalizado, o sea cuando se aplica una serie de castigos basados en la suspensión de algunos derechos alienables a las personas naturales. De este modo, una persona es reincidente cuando, habiendo sido penalizada por un delito, ella vuelve a cometer otra conducta desviada tipificada como delito. De tal forma que, para configurar la reincidencia tiene que haber a lo menos dos acciones ilícitas comprobadas, separadas por un tiempo delimitado y habiendo recibido los estímulos por parte del Estado, para no volver a cometer ilícitos. Es posible así, distinguir reincidencia de reiteración: la reiteración es una suma de acciones delictivas, las que, si bien están necesariamente separadas por espacios temporales, entremedio de ese espacio temporal no existe un estímulo estatal como cancelación de algunos derechos para frenar las acciones delictivas. 
Al mismo tiempo, es preciso distinguir reincidencia de reencarcelamiento: el reencarcelamiento puede ser entendido como la situación en que una persona ha sido ingresada dos o más veces al interior del sistema penitenciario, sin hacer referencia a su estado procesal, o sea puede haber sido condenada anteriormente y estar actualmente imputada, o dos veces imputada. Solo la situación procesal de dos o más condenas ejecutoriadas se considera como reincidencia. Una cuestión relevante aquí, desde el punto de vista de la socialización criminal o de la estigmatización social asociada a la cárcel es la siguiente: el hecho de estar condenado a un año de cárcel o estar el mismo tiempo en detención preventiva no debiera tener diferencias significativas, sobre todo en las cárceles latinoamericanas, donde el problema de la sobrepoblación atenta contra cualquier intento de segmentación interna efectiva que sea consistente con el objetivo de la prevención de la socialización criminal.

En el siguiente diagrama se pueden observar bastante bien las consideraciones esquemáticas mínimas de la reincidencia, es decir, una serie de nociones que nos permiten ordenar la discusión: la condena de referencia, el tiempo de ventana y el reingreso por nueva condena.

\section{ESQUEMA 1}

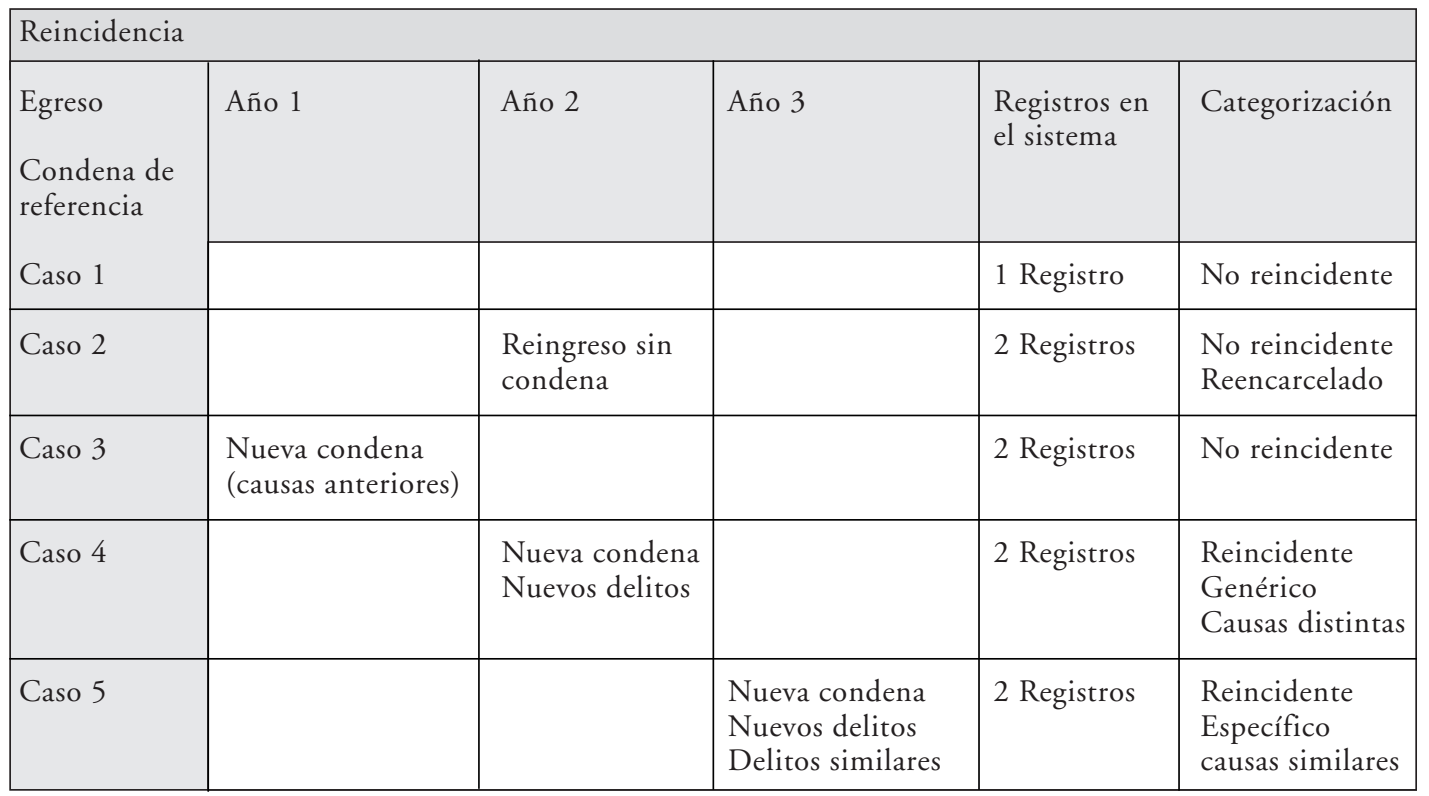

Este esquema expone, pues, situaciones típicas en el proceso de constitución de la reincidencia. El primer caso muestra a un no reincidente con solo un registro de egreso; el segundo alude a un reencarcelado típico, o sea, una persona con dos registros al interior del sistema, pero solo uno de ellos corresponde a una causa ejecutoriada, tratándose aquí de un no reincidente; el tercer caso corresponde a una persona con dos registros, pero al 
momento de egresar de una causa comienzan a correr nuevas condenas derivadas de causas anteriores no cerradas, en este caso un "no reincidente" en términos de las ciencias sociales, pero sí reincidente para el sistema jurídico. El caso cuatro nos ilustra respecto de un reincidente típico por cumplimiento, donde las dos causas están ejecutoriadas, separadas por un tiempo de ventana, aunque la diferencia con el caso cinco es que en el caso cuatro los delitos son de distinta especie.

De este modo, podemos abordar la noción de reincidencia desde la perspectiva de las ciencias sociales, dando cuenta del conjunto de acciones que deben realizarse para que se considere reincidente a un interno y por qué. Así, la reincidencia queda entendida desde la óptica de las ciencias sociales como la condición de haber sido condenado por una causa cuando existe otra condena anterior ejecutoriada. Obsérvese que no se trata simplemente de dos delitos, sino de dos causas de ingreso asociadas a uno o a varios delitos, las que están en estado de ejecutoriadas.

\section{EXPERIENCIA DE MEDICIÓN DE LA REINCIDENCIA EN EL SISTEMA PENAL CHILENO}

Como antecedente se tiene un estudio realizado en 1999 por la Unidad de Investigaciones Criminológicas de Gendarmería de Chile, en que el universo lo constituyó la población de condenados egresados durante el primer semestre de 1993, atendida por GENCHI, aplicando en el conteo de casos el concepto de reincidencia legal, que definió como reincidentes a quienes han sido condenados más de una vez por los tribunales de Justicia. También existe otro trabajo anterior referido a la experiencia desarrollada solo con medidas alternativas a la reclusión. A continuación se muestra una reseña del resultado de ambos estudios.

\section{TABLA 1}

Estudio de Reincidencia para el Medio Libre. 1996

10 Estudio (1996):

\begin{tabular}{|c|c|c|c|c|c|}
\hline Medida & $\begin{array}{c}1^{\mathrm{er}} \text { cuatrimestre } \\
\text { de egreso }\end{array}$ & $\begin{array}{l}\text { Universo } \\
\text { egresados }\end{array}$ & $\begin{array}{l}\text { Muestra } \\
\text { egresados }\end{array}$ & $\begin{array}{c}\text { Error } \\
\text { muestral }\end{array}$ & $\begin{array}{l}\text { Tasa } \\
\text { reincidencia }\end{array}$ \\
\hline \multirow[t]{3}{*}{ Libertad Vigilada del Adulto } & 1990 & 78 & 42 & $11 \%$ & $16.6 \%$ \\
\hline & 1991 & 121 & 114 & $2 \%$ & $13.1 \%$ \\
\hline & 1992 & 141 & 89 & $6 \%$ & $6.7 \%$ \\
\hline Total & & 340 & 245 & $3 \%$ & $11.4 \%$ \\
\hline Reclusión Nocturna & 1990 & 469 & 192 & $6 \%$ & $19.8 \%$ \\
\hline Remisión Condicional de la Pena & 1990 & 4167 & 235 & $6 \%$ & $9.8 \%$ \\
\hline
\end{tabular}

Fuente: Estudio reincidencia (2005-2006). UNICRIM. 
El estudio de 1996 trabajó sobre el sistema de tratamiento en el medio libre, tomando muestras representativas de cada una de las medidas de tratamiento. Se constató un $16,6 \%$ de reincidencia en la libertad vigilada del adulto, un 19,8\% de reincidencia para la reclusión nocturna y un 9,8\% para la remisión condicional de la pena. Uno de los problemas de esta investigación es que toma el año de egreso a 1990, pero no hay claridad en el año en que mide la reincidencia, en tanto nueva condena, por lo cual no logra medir la tasa según períodos de ventana temporal. Además, solo considera muestras del primer cuatrimestre de egreso, por lo tanto se producen alteraciones en la medición anual, ya que no es posible asumir el total de egresados del año. Estos dos elementos resultarán centrales en los posteriores estudios.

TABLA 2

Estudio de Reincidencia. 1999

\begin{tabular}{lccccc}
\hline 20 Estudio (1999): & & & & & \\
Sistema o Medida & $\begin{array}{c}\text { er semestre } \\
\text { de egreso }\end{array}$ & $\begin{array}{c}\text { Universo } \\
\text { de egreso }\end{array}$ & $\begin{array}{c}\text { Muestra } \\
\text { egresados }\end{array}$ & $\begin{array}{c}\text { Error } \\
\text { muestral }\end{array}$ & $\begin{array}{c}\text { Tasa } \\
\text { reincidencia }\end{array}$ \\
\hline Privativo de Libertad (Intramuros) & 1993 & 1972 & 380 & $5 \%$ & $47.4 \%$ \\
Libertad Vigilada del Adulto & 1993 & 226 & 168 & $5 \%$ & $16.1 \%$ \\
Reclusión Nocturna & 1993 & 488 & 232 & $4 \%$ & $20.3 \%$ \\
Remisión Condicional de la Pena & 1993 & 4164 & 545 & $4 \%$ & $6.4 \%$ \\
\hline
\end{tabular}

Fuente: Estudio reincidencia (2005-2006). UNICRIM.

El segundo trabajo corresponde a la medición realizada en 1999, otra vez trabajando con muestras y tomando casi seis años de ventana, entre la toma del año de referencia y los años de reingreso con condena. Este elemento es el que explica el alto porcentaje de reincidencia que, como vemos, marca para una muestra de 380 casos 47,4\% de personas que ya registraban una condena. Un elemento que explica esta alza es el efecto de tasa acumulada que se produce cuando se mide la reincidencia con tantos años de ventana. Esto significa que, al tomar un año de referencia frente a un año de reingreso o un espacio de tiempo variable entre condenas, se produce una acumulación de casos durante ese tiempo que registran dos condenas, resultando estos elementos la base para construir la tasa, ya que solo es el número de casos reingresados frente a un $\mathrm{N}$ total de egresados. Así, curiosamente, se toma un tipo de tasa temporal acotada, ya que no es capaz de determinar los ritmos de crecimiento, principal condición de cualquier estudio de tendencia o que asuma al tiempo como elemento central.

El problema de los ritmos de crecimiento resulta relevante, porque una cuestión elemental es que a medida que se avanza en los años pueden volver a reingresar los seleccionados de la muestra haciendo que la tasa aumente hasta un cierto límite, que tampoco se puede determinar. Una forma de no caer en este error metodológico es tomar al total de reingresados con condena sobre el total de egresados del período, sin fijar un 
año como base de la ventana. Esta es la que se puede denominar tasa general de reincidencia; esta tasa general de reincidencia establece el total de egresados durante un período frente al total de reingresado del mismo período, sin tomar como referencia al año en tanto unidad descriptiva ni al tiempo como evolución, sino como un referente de un espacio temporal como período. Un elemento importante de esta tasa general es que permite nominalizar posteriormente la etapa de ventana, y determinar qué tipo de composición tiene la población penal en un año o periodo y cuál de estos aporta mayor cantidad de reincidentes a la tasa general. De esta forma, un año específico se vuelve objeto de investigación, como, por ejemplo, el que haya sido un período de entrada en vigencia de una determinada ley, mientras en el otro modelo esto no es posible realizar.

En el estudio realizado en el año 2005 se aprecian algunos ajustes al interior del cálculo de la tasa de reincidencia, incorporando algunas de las críticas esbozadas, aunque no de manera explícita.

En el siguiente grafico, que corresponde a la investigación del 2005, vemos como se pierde la idea de tasa general de reincidencia, y se procede por medio de otro tipo de cómputo, el que presenta una serie de problemas metodológicos insalvables hasta ahora, porque no se fijan los universos de cálculo.

\section{GRÁFICO 1}

\section{Tasa de reincidencia intramuros. 2006}

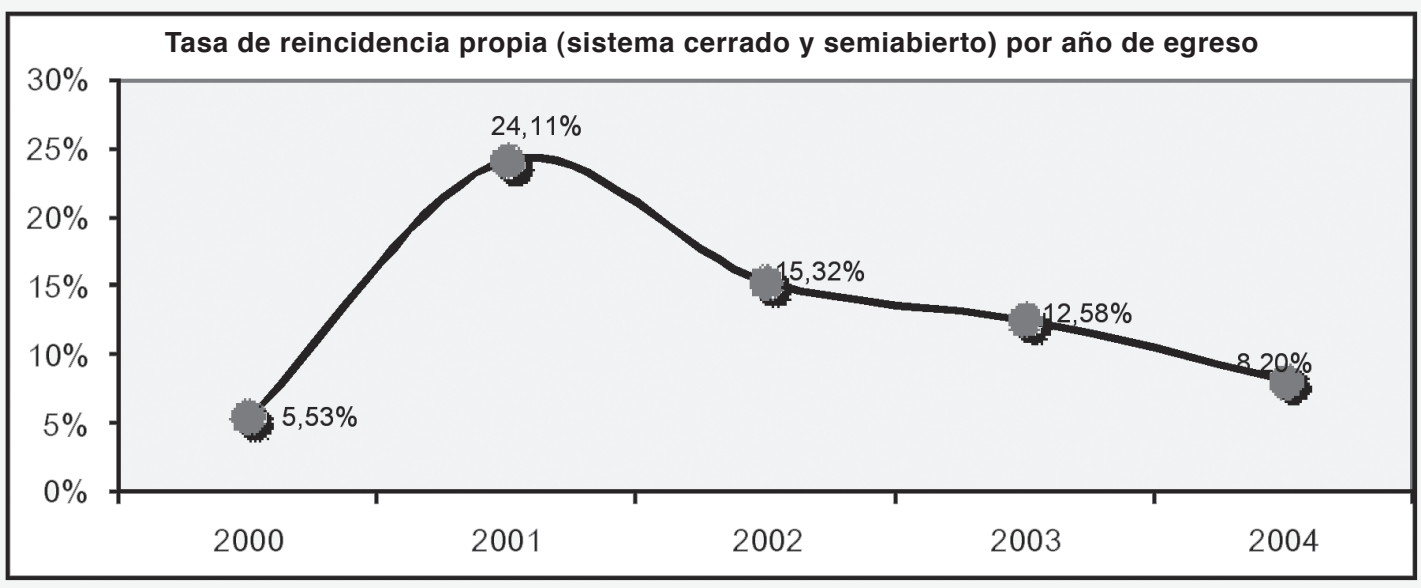

Fuente: Estudio reincidencia (2005-2006). UNICRIM.

Vemos en el gráfico que en el 2001 se registra el porcentaje más alto de reincidencia, lo que implica que ese año es el que contribuye con mayor cantidad de egresados/ reingresados durante el período de estudio, calculado sobre el total de egresados frente a los reingresados. Este elemento, nótese, no corresponde a la tasa de reincidencia del año 2001, sino solo a la participación del año en cuestión dentro del total de egresados/ reingresados, lo que genera una distorsión en las cifras, ya que si los estudios anteriores sufren de tasa acumulada, este nos impide saber la tasa general de reincidencia, pues oculta 
la tasa general acumulada del periodo y solamente presenta las participaciones de ciertos años durante el periodo. De hecho, al sumarse obtenemos una cifra de 65,74\% de reincidencia general acumulada, lo cual demuestra que se calculó sobre el total de egresados/ reingresados y no sobre los reincidentes específicamente. Un modo de subsanar esto es calcular la tasa de reincidencia acumulada del período, y luego comparar los años que más aportan a esta tasa dentro de un nuevo universo que serían los reincidentes detectados, como ya se esbozó anteriormente.

En las tablas que se presentan a continuación es posible apreciar que el fenómeno se sigue repitiendo: no sabemos la tasa general del período y no se conoce el aporte de los años sobre el universo de reincidentes. Este tipo de metodología debe ser criticado con el objetivo de obtener mejores observaciones sobre las tasas de reincidencia. Y debe completarse con mediciones que trabajen en base a otro tipo de metodologías, que no sea el recuento de casos en espacios temporales discretos, sin saber las referencias y los universos contemplados.

\section{TABLA 3}

Tasa de reincidencia por sexo. 2006

\begin{tabular}{lccccc}
\hline $\begin{array}{l}\text { Tasa de reincidencia según sexo } \\
\text { por año de egreso }\end{array}$ & 2000 & 2001 & 2002 & 2003 & 2003 \\
\hline Hombres & $16,67 \%$ & $18,46 \%$ & $8,65 \%$ & $16,44 \%$ & $15,06 \%$ \\
Mujeres & $5,26 \%$ & $24,33 \%$ & $15,60 \%$ & $12,39 \%$ & $7,77 \%$ \\
Total & $5,53 \%$ & $24,11 \%$ & $15,32 \%$ & $12,58 \%$ & $8,20 \%$ \\
\hline
\end{tabular}

Fuente: Estudio reincidencia (2005-2006). UNICRIM.

\section{LOS RITMOS DE CRECIMIENTO DE LA REINCIDENCIA}

La tabla precedente revela que es posible calcular la tasa de reincidencia por medio de encuestas y expone los resultados de una realizada a sujetos de entre 18 y 30 años, con muestras representativas de grupos quinquenales de edad, o sea, grupos definidos entre 15-19, 20-24 y 25-29 años. Realizada durante 2006, la encuesta incluyó una pregunta precisa: ¿Esta es su primera condena?; interrogante que al ser respondida en negativo, permite construir la variable reincidencia cuando la recodificamos. También se preguntó sobre el ingreso en la última condena y el egreso anterior en la penúltima condena, considerando así un lapso que permite dar cuenta de un período que no hace referencia a años en tanto nominalidad de los años, sino a un espacio temporal entre condenas. Como vemos, la tasa durante un período de nueve años como máximo y 0 año como mínimo, por efecto de la edad de los entrevistados, nos da una tasa general acumulada del 31,96\%; en esta cifra se aprecia el efecto de tasa acumulada que habíamos anticipado. 
TABLA 4

Tasa de reincidencia acumulada y evolución de crecimiento de la tasa

\begin{tabular}{|c|c|c|c|c|c|c|c|c|c|c|c|}
\hline \multicolumn{7}{|c|}{ Tasa de reincidencia acumulada y evolución de crecimiento de la tasa } \\
\hline 0 & 9,38 & 9,38 & & & & & & & & & \\
\hline 1 & & 20,31 & 10,93 & & & & & & & & \\
\hline 2 & & & 24,57 & 4,26 & & & & & & & \\
\hline 3 & & & & 26,42 & 1,85 & & & & & & \\
\hline 4 & & & & & 29,7 & 3,27 & & & & & \\
\hline 5 & & & & & & 30,8 & 1,13 & & & & \\
\hline 6 & & & & & & & 31,39 & 0,57 & & & \\
\hline 7 & & & & & & & & 31,68 & 0,29 & & \\
\hline 8 & & & & & & & & & 31,82 & 0,14 & \\
\hline 9 & & & & & & & & & & 31,96 & 0,14 \\
\hline
\end{tabular}

Fuente: Elaboración propia desde Encuesta 2006 UNICRIM.

Calcular la variación interanual de la tasa acumulada permite mostrar que los ritmos de crecimiento son absolutamente disímiles; de esta manera, el máximo de crecimiento de una tasa acumulada de reincidencia se alcanza a los dos años de egreso, y luego el crecimiento se detiene sistemáticamente, aunque la tasa de reincidencia sigue aumentando. En esta tabla se observa que hay cierta fragilidad en las acciones de los sujetos al momento de egresar; así, mientras más cercano está el egreso, la probabilidad de reincidir se hace más alta y al revés, mientras existe mayor distancia la probabilidad decrece profundamente. De esta forma, podemos constatar que entre los tres primeros años hay un $25 \%$ de probabilidad de reincidencia para los grupos más jóvenes, aquellos de entre los 15 y 29 años, descendiendo abruptamente una vez superado el tercer año de egreso. Un elemento sensible de la reincidencia al parecer resulta ser la edad de iniciación en las actividades delictivas y la edad al momento del ingreso.

El siguiente gráfico ofrece diferencias de medias. El grupo más joven, de entre 1519 años, informa que son las personas que permanecen menos tiempo en el medio libre postcondena de referencia, y que este tiempo va aumentando en la medida que subimos en los rangos de edad, siendo de este modo significativo el modelo. Este elemento no deja de ser preocupante a la luz de la nueva ley de responsabilidad juvenil, pues muestra que en la medida que las personas son más jóvenes al ingreso, sus probabilidades de reingreso se hacen más altas; ello genera un efecto en cadena, pues provocará que sean parte del grupo que persistirá en sus actividades delictivas y con eso persistirán también sus riesgos de volver a la cárcel, con penas más largas. 


\section{GRÁFICO 2}

Tiempo transcurrido en el medio libre de población reincidente por estrato etario

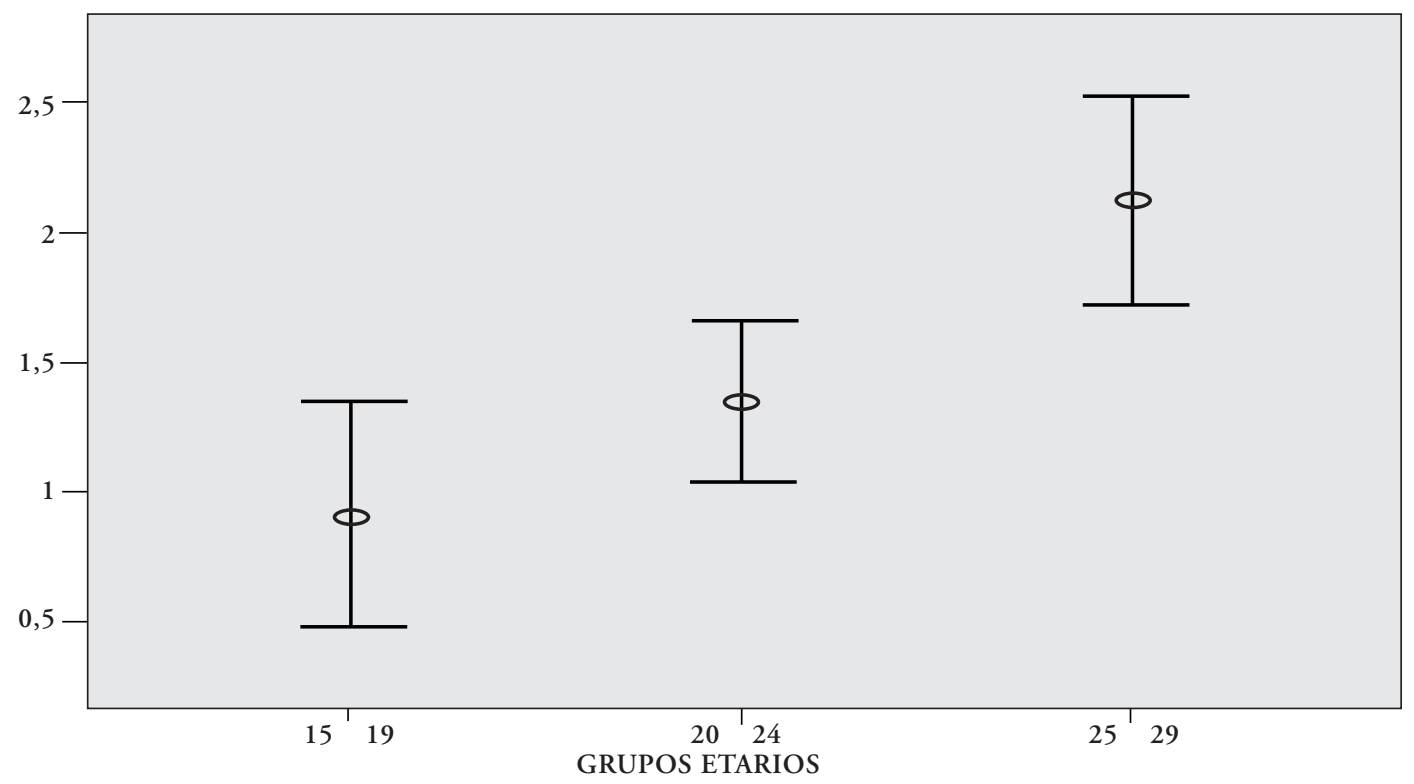

Fuente: Elaboración propia desde Encuesta 2006 UNICRIM

Esta condición hace pensar en cierto remanente de población que se va quedando al interior del sistema penitenciario, o que entra y sale del sistema, generando efectos sobre el trabajo de reinserción social y sobre la socialización intrapenitenciaria. En estos remanentes de población es donde la reincidencia marca con fuerza el índice de compromiso delictual; son estos grupos los considerados dentro de un ciclo penal en que la cárcel se enlaza con los modos de vida, al punto de hacer que los núcleos familiares se adapten al proceso de entrada y salida del sistema, y nuevos miembros de la familia ingresen al mismo. Un indicador de ello deriva de la pregunta por los miembros del núcleo familiar respecto de si están o han estado al interior del sistema penal, la cual dio como resultado un $40 \%$, al momento en que se interrogó a las mujeres al interior del sistema durante el año $2005^{1}$. Por lo tanto, podemos ver la relación del sistema penal con los grupos familiares de algunos condenados, quienes tienden a enlazarse sistemáticamente con el mundo penitenciario.

\section{UNA PROPUESTA DE MODELO DE MEDICIÓN DE REINCIDENCIA}

El siguiente esquema puede contribuir a lograr una solución a la serie de interrogantes planteadas en los estudios anteriores: a) la tasa general de reincidencia, b) los períodos

1 AEDO, Andrés. "La reproducción social de la cárcel: unidad doméstica, ciclo penal y cuestión social". En: Revista Estudios criminológicos y penitenciarios. Número 9. Chile. ISSN 0717-5744. Diciembre 2006. 
de medición y pesos relativos de cada año nominal en el período, c) los efectos de tasa acumulada, y d) los ritmos de evolución de la reincidencia. Se trata, entonces, de componentes centrales de la idea de reincidencia en términos metodológicos, que se abren para mostrar las distinciones que podemos hacer con ellos. Estos cuatro indicadores de reincidencia, que se desprenden de la crítica de los estudios anteriores, constituyen, pues, una base para elaborar un modelo que incorpore a todos los indicadores posibles de construir.

De esta manera es posible proponer un modelo integrado de medición de la reincidencia, aunque en este punto debemos volver sobre nuestros conceptos esbozados al principio, pero esta vez delimitados: a) causa de referencia (condenado/no condenado), b) causa de reingreso (condenado/ no condenado), c) ventana de tiempo, y d) años nominales. Si simbolizamos por medio de la letra $\mathrm{T}$ al tiempo, ella nos puede delimitar, entre $\mathrm{T} 1$ y T2, el período temporal en tanto años de ventana, e, igualmente, al nominalizarse en años discretos, nos mostrará el año de referencia considerado para la medición. En el momento en que se decida proceder al cálculo de la tasa de reincidencia, se deben incorporar las diferentes tasas, que por medio del modelo resultan: la tasa general de reincidencia de un período en tanto tasa general acumulada, donde se toma todo el universo de egresados sin referencia a un año nominal, sino a un período entre años frente a todos los reingresados con condena. Distinta resulta la tasa acumulada de reincidencia con años de ventana establecidos desde el año de referencia, donde se toma como universo a todos los egresados de un año frente a todos los condenados de los años siguientes, dependiendo del período de ventana que se estime conveniente medir. En referencia a los cuadrantes de abajo, hace posible asimismo calcular los aportes de los años nominales, en tanto ritmos de crecimiento de las participaciones de los años respecto a la tasa general de reincidencia y a la tasa de reincidencia considerando año de referencia.

\section{ESQUEMA 2}

Modelo integrado de medición de reincidencia

\begin{tabular}{|c|c|c|c|c|c|c|}
\hline \multicolumn{7}{|l|}{ Tiempo de egreso } \\
\hline Años & $\mathrm{T}-4$ & $\mathrm{~T}-3$ & $\mathrm{~T}-2$ & $\mathrm{~T}-1$ & $\mathrm{~T}$ & \\
\hline \multirow[t]{6}{*}{ Periodo temporal } & 0 & 0 & 0 & 0 & 0 & Tasa acumulada general \\
\hline & & 1 & 2 & 3 & 4 & Tasa acumulada 4 años de ventana \\
\hline & & & 1 & 2 & 3 & Tasa acumulada 3 años de ventana \\
\hline & & & & 1 & 2 & Tasa acumulada 2 años de ventana \\
\hline & & & & & 1 & Tasa anual \\
\hline & $\begin{array}{l}\text { Tasa } \\
\text { anual }\end{array}$ & $\begin{array}{c}\text { Tasa } \\
\text { anual } 1\end{array}$ & $\begin{array}{c}\text { Tasa } \\
\text { anual } 2\end{array}$ & $\begin{array}{c}\text { Tasa } \\
\text { anual } 3\end{array}$ & $\begin{array}{c}\text { Tasa } \\
\text { anual } 4\end{array}$ & $\begin{array}{l}\text { Ritmo de crecimiento general } \\
\text { Descomposición del ritmo por año }\end{array}$ \\
\hline
\end{tabular}

Fuente: elaboración propia. 
Creemos, en consecuencia, que podemos calcular la tasa de reincidencia lo más limpia posible, contemplando los efectos de la tasa acumulada general o tasas con referencia temporal vacía, toda vez que incorporaremos estos efectos en la metodología de cálculo. Por consiguiente, se puede proceder al cálculo de las tasas generales de reincidencia, a la tasa acumulada con año de referencia y a las tasas de participación anual en ambas, la tasa de crecimiento anual de la reincidencia en tanto ritmo de crecimiento en períodos temporales abstractos. Así, podremos dar cuenta del total de los reincidentes frente al total de los ingresados en un período, pudiendo observar con gran detalle la composición de la población penal y la prevalencia de la reincidencia en el sistema.

Ahora bien, ¿cuál es la tasa de reincidencia, si hay tantas y que pueden arribar a resultados tan distintos? Sería incongruente establecer una a través de un promedio de las tasas, cuando por el contrario, el punto central es que las tasas de reincidencia se deben utilizar en varios niveles distintos, donde podemos distinguir el metodológico y el interpretativo. Ejemplo del primero son las ya nombradas: tasa general del período, tasa de crecimiento del período, tasa de participación de un año nominal en el período, tasa de reincidencia imputable a un año nominal, tasa de crecimiento de reincidencia. Existe, pues, una batería de indicadores que pueden resultar útiles para elaborar subproductos, como la tasa de reincidencia de las personas afectadas por un programa específico, donde la plasticidad de los indicadores nos puede hacer avanzar hacia estudios cuasi experimentales. Consideramos que la complejidad que caracteriza a la tasa de reincidencia se explica en la medida de que ella adopta distintas formas, con lo que la fijación de esta polisemia debe realizarse por medio del nivel interpretativo de los variados indicadores existentes. El punto principal radica, entonces, no solo en el tratamiento de los datos, sino también en cómo ellos se incorporan al sistema penal y al de justicia, cuestión que se aborda en el último subtítulo de este estudio.

\section{PROPUESTA DE MODO DE EVALUACIÓN DE LA REINCIDENCIA}

Como hemos advertido, abordamos ahora al último elemento de discusión. No hemos quitado variación al concepto, aunque lo hemos delimitado un poco más, aunque aquello que se ganó en teoría, elaborando una distinción, se perdió en metodología. La misma distinción generó nuevas variantes de sí misma, pero aquí ya no hay polisemia, sino complejidad. La tarea pasa ahora por reducir esta complejidad, por medio de una nueva selección. Aquí, solo la interpretación de los indicadores de reincidencia puede darnos salidas para nuestro nuevo problema. Esto no resulta fácil, porque las preguntas se pueden multiplicar.

Si bien el sistema penal tiene como misión resocializar, y por lo tanto evitar la prevalencia de la reincidencia en la composición de la población penal, no resulta del todo sencillo conocer cuáles son las cifras relevantes para el sistema penal y para el resto de los sistemas punitivos y de protección penal. Las preguntas pueden formularse de la siguiente manera: ¿Hasta dónde puede y debe hacerse cargo el sistema penitenciario de las condiciones de sus egresados? ¿En qué momento terminan las responsabilidades del sistema estatal? 
¿Cuándo comienzan las dinámicas sociales de los mercados de trabajo y de otros sistemas a funcionar? ¿Las condiciones de los egresados dependen solamente de ellos o hay una zona mixta de trabajo pendiente con los penados? ¿Las posibles altas tasas de reincidencia son una muestra del fracaso de la política punitiva nacional?

Una buena forma de trabajar este punto es la distinción entre agencia / estructura y los modos en que esta distinción genera temporalidad (Archer, 1995). Podemos decir que el sistema de justicia trabaja con el fetichismo de la estructura y el sistema de seguridad con el fetichismo de la agencia, donde el primero destaca las condiciones de vulnerabilidad social pre y postinstitucional de los penados; y el segundo, las condiciones de resiliencia moral frente a la vulnerabilidad social pre y postinstitucional. En el primer caso se cancelarán o eludirán las condiciones del agente, mientras el segundo hará otro tanto con la estructura. Esto quiere decir que se apuesta a los impactos que tenga la política de justicia frente a los agentes, con la esperanza de que no vuelvan a cometer delitos con una condición de responsabilidad estatal permanente; la segunda dejará completamente la responsabilidad a los agentes como actores racionales que calculan la utilidad de su propia trayectoria de vida, teniendo sentido la condición asistencialista en la primera y la respuesta punitiva en la segunda. Ambos modos de comprensión del fenómeno delictivo y del encarcelamiento penal tienen el vicio de contraer el problema social de la delincuencia a las estructuras o a los agentes, cayendo ambas en paradojas incontrolables, ya que se llenan de conclusiones que no les permiten distinguir a tiempo y con el tiempo. En el primer caso, como ya advertimos, la responsabilidad es total en el tiempo; y en el segundo, simplemente no hay tiempo de responsabilidad, porque es un problema de decisiones personales.

Según la teoría que estamos haciendo alusión, la estructura siempre es primero, aunque a pesar de las condiciones teóricas del origen, la estructura es siempre anterior y la agencia posterior. Esto implica que debe haber un balance entre las condiciones estructurales del agente y las condiciones agenciales de la estructura, en la producción de tiempo en el medio libre. Estos elementos permiten establecer límites temporales donde se espera que la estructura institucional vaya generando impacto en los agentes, y que luego esos impactos logren generar modificaciones de los agentes en los modos de inserción en la estructura. Esto implica lo siguiente: es central modificar las posiciones de los agentes en la estructura social, por ejemplo, las condiciones de inserción laboral de las personas. Las capacitaciones pertinentes son absolutamente relevantes, para que las personas puedan modificar su inserción en la estructura, es decir modificar las habilidades, competencias y conocimientos, para que el ciclo que los llevó al sistema penal no se reproduzca. Junto a ello es preciso que los agentes puedan -al estar modificadas sus propiedades como modos de intercambio con el sistema económico- construir una inserción laboral con mayores retornos monetarios.

La pregunta siguiente es: ¿cuánto tiempo implica esto? El tiempo de ventana necesario entre las condenas de referencia y la condena de reingreso, que es también tiempo de ventana en el medio libre. Si consideramos tres años como el punto en que se alcanza el máximo de probabilidad de reingreso, para luego caer abruptamente en porcentajes menores, ese mismo tiempo de ventana es el que debe ser incorporado por parte del sistema de 
justicia, con el propósito de modificar las condiciones estructurales de la inserción. El supuesto es aquí que los agentes ya disponen de nuevas propiedades con las cuales mejorar su inserción. Este elemento genera las siguientes distinciones: la responsabilidad del sistema penal llega hasta el límite de tres años y luego comienza la responsabilidad de los agentes. Esto hace que varíen los modos en que el sistema se autoobserva, por medio de la medición de la reincidencia, sin caer en la trampa estructural, y sin caer tampoco en la trampa agencial, dándole tiempo a cada una para que puedan interactuar, generando cambios en el agente frente a la estructura. Todo esto trae aparejado el problema del trabajo de preparación para el egreso y post penitenciario, el cual debe ser concebido con el objetivo de evitar la reincidencia, no solo en base a la ya nombrada resiliencia moral, sino en un plano de cambio de las capacidades agenciales de intercambio con los sistemas con los que tranza prestaciones; además, es indispensable sumar esfuerzos institucionales entre lo intra y lo extrapenitenciario.

A partir del modelo de medición, lo que proponemos en todo caso es un modo de interpretación de ingreso de datos al interior del sistema. De esta forma, la variación más profunda estará radicada en los códigos con que se leerán las cifras de reincidencia. Por ejemplo, en un modelo tipo dicotómico (código binario), se puede entender la reincidencia como un suceso A y la no reincidencia como un suceso B, donde A es fracaso y B éxito. Sin embargo, si proponemos al tiempo como elemento de variación de los modos de responsabilidad, la interpretación también cambia. Así, podemos decir -y queda bastante claro en el esquema posterior- que el suceso A solo puede ser considerado en un límite de tres años, por lo que sí tiene ocurrencia más allá de ese período, si bien es reincidente, la interpretación es distinta, ya que se considera un elemento propio del agente, por lo tanto constituye éxito. En cambio, si la reincidencia ocurre como suceso antes del periodo mencionado, debe ser incorporado como fracaso.

\section{ESQUEMA 3}

Modelo de interpretación de la reincidencia para el sistema penal

\begin{tabular}{|c|c|c|c|c|c|c|}
\hline \multicolumn{7}{|l|}{ Reincidencia } \\
\hline $\begin{array}{l}\text { Egreso } 1^{\circ} \\
\text { registro causa }\end{array}$ & Año 1 & Año 2 & Año 3 & Año 4 & Condicion & Evaluación \\
\hline Caso 1 & & & & & No reincidente & Éxito \\
\hline Caso 2 & & & $\begin{array}{c}\text { Reingreso } \\
\text { sin condena }\end{array}$ & & $\begin{array}{l}\text { No reincidente } \\
\text { Reencarcelado }\end{array}$ & Fracaso \\
\hline Caso 3 & $\begin{array}{l}\text { Causa anterior } \\
\text { Nueva condena }\end{array}$ & & & & No reincidente & Éxito \\
\hline Caso 4 & & $\begin{array}{l}\text { Nueva condena } \\
\text { Nuevos delitos }\end{array}$ & & & $\begin{array}{c}\text { Reincidente } \\
\text { Responsabilidad } \\
\text { estructural }\end{array}$ & Fracaso \\
\hline Caso 5 & & & & $\begin{array}{l}\text { Nueva condena } \\
\text { Nuevos delitos }\end{array}$ & $\begin{array}{l}\text { Reincidente } \\
\text { Responsabilidad } \\
\text { agencial }\end{array}$ & Éxito \\
\hline
\end{tabular}

Fuente: elaboración propia. 
Este modo de pautar la incorporación de cifras mediante un código, facilitará identificar el impacto del sistema carcelario, pudiendo a posteriori, simplificar los estudios y disponer de un indicador duro sobre el modo en que el sistema penal y sus políticas tienen efectos sobre los internos. Pero, por sobre todo, ofrece la ventaja de poder construir un modo de interpretación sistémica propio de las cifras de los estudios, dando cuenta de la posibilidad de tomar una forma dentro del sistema de justicia, que pueda transmitir comunicaciones al resto de los sistemas sociales, y no caer en la constante recepción de políticas, estudios y visiones novedosas sin tener la posibilidad de incorporarlos de manera eficiente y eficaz a un sistema ya estructurado.

\section{REFERENCIAS BIBLIOGRÁFICAS}

ARCHER, Margaret. Realist Social Theory: the Morphogenetic Approach. Cambridge University Press. UK, 1995.

BaratTA, Alessandro. Criminología crítica y critica del derecho penal. México, Siglo XXI, 1990.

BAUman, Zygmunt. En busca de espacio público. Argentina, FCE, 1999.

Gendarmería de Chile. “Complejo penitenciario de Punta Arenas: estudio cualitativo exploratorio del cambio de recinto penal de Punta Arenas”. Documento interno. Gendarmería de Chile. 2005.

Aedo Henríquez, Andrés. "La reproducción social de la cárcel: unidad doméstica, ciclo penal y cuestión social" en: Revista de Estudios Criminológicos y Penitenciarios N 9, 2006.

Hernández Sampieri, Roberto, Fernández Collado, Carlos y Baptista Lucio, Pilar. Metodología de la Investigación. México, Mc Graw Hill, 2003.

LACLAU, Ernesto. Emancipación y Diferencia. Buenos Aires, Ariel, 1996.

LAKATOS, Imre. La metodología de los programas de investigación científica. España, Alianza, 1983.

LARRAURI, Larrauri. La herencia de la criminología crítica. España, Siglo XXI, 1992.

LUHMANN, Niklas. Complejidad y modernidad: de la unidad a la diferencia. Madrid, Trotta, 1998.

Olin Wright, Erik. The Politics of Punishment: A Critical Analysis of Prisons in America. New York: Harper and Row and Harper Colophon Books, 1973. http://www.ssc.wisc.edu/ - wright

PARdinas, Felipe. Metodología y técnicas de investigación en ciencias sociales. México, Siglo XXI, 1991.

UNICRIM, Investigadores. Tasas de reincidencia de condenados egresados del sistema penitenciario. Cuaderno N² UNICRIM. Chile. 2000.

WACQUANT, Löic. Las cárceles de la miseria. Argentina, Manantial, 2000. 
